\title{
Article
}

\section{An account of subjective probability judgment for joint events: Conjunctive and disjunctive}

Fisk, John, Marshall, Dean, Rogers, Paul and Stock, Rosemary

Available at http://clok.uclan.ac.uk/28455/

Fisk, John ORCID: 0000-0002-2981-0870, Marshall, Dean, Rogers, Paul and Stock, Rosemary (2019) An account of subjective probability judgment for joint events: Conjunctive and disjunctive. Scandinavian Journal of Psychology, 60 (5). pp. 405-420. ISSN 0036-5564

It is advisable to refer to the publisher's version if you intend to cite from the work. http://dx.doi.org/10.1111/sjop.12560

For more information about UCLan's research in this area go to http://www.uclan.ac.uk/researchgroups/ and search for <name of research Group>.

For information about Research generally at UCLan please go to http://www.uclan.ac.uk/research/

All outputs in CLoK are protected by Intellectual Property Rights law, including Copyright law. Copyright, IPR and Moral Rights for the works on this site are retained by the individual authors and/or other copyright owners. Terms and conditions for use of this material are defined in the policies page.

\section{CLoK}

Central Lancashire online Knowledge www.clok.uclan.ac.uk

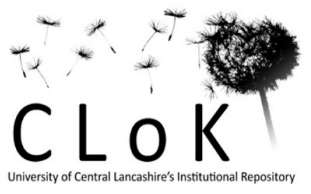


An account of subjective probability judgement for joint events: Conjunctive and Disjunctive

John E. Fisk ${ }^{\mathrm{a}}$; Dean A. Marshall ${ }^{\mathrm{a}}$, Paul Rogers ${ }^{\mathrm{b}}$, Rosemary Stock $^{\mathrm{c}}$

a: School of Psychology, University of Central Lancashire, Preston PR1 2HE, UK

b :Department of Psychology, Goldsmiths College, University of London, New Cross, SE14 6NW, UK

c: School of Human and Social Sciences, University of West London, Brentford, Middlesex TW8 9GA, UK

Running Head: Probability judgement for joint events

Corresponding author:

Professor John E Fisk, Ph D

School of Psychology

University of Central Lancashire

Preston PR1 2HE

United Kingdom

Tel 44 (0) 1772894465

Fax 44 (0) 1772892925

e-mail: jfisk@uclan.ac.uk

Word Count: 12903 (excluding abstract, references \& tables) 


\begin{abstract}
Probability judgement is a vital part of many aspects of everyday life. In the present paper, we present a new theory of the way in which individuals produce probability estimates for joint events: conjunctive and disjunctive. We propose that a majority of individuals produce conjunctive (disjunctive) estimates by making a quasi-random adjustment, positive or negative, from the less (more) likely component probability with the other component playing no obvious role. In two studies, we produce evidence supporting propositions that follow from our theory. First, the component probabilities do appear to play the distinct roles we propose in determining the joint event probabilities. Second, contrary to probability theory and other accounts of probability judgement, we show that the conjunctive-less likely probability difference is unrelated to the more likely- disjunctive probability difference (in normative theory these quantities are identical). In conclusion, while violating the norms of probability judgement, we argue that estimates produced in the manner we propose will be close enough to the normative values especially given the changing nature of the external environment and the incomplete nature of available information.
\end{abstract}

Key Words: conjunction, disjunction, fallacy, probability judgement 
Over the last 50 years or more, a body of evidence has accumulated demonstrating that individuals violate some of the key normative rules of probability theory (Kahneman, 2016). Erroneous probabilistic judgements may have potentially serious consequences. For example, regarding Bayesian judgements, failure to take account of likelihood of a false positive outcome might result in undue weight being attached to the result of a diagnostic test. Furthermore, violations of the disjunction and conjunction rules have been shown to result in biased judgements in the domains of social cognition and medical diagnosis. For example, conjunctions of personal attributes (e.g., thoughtful and intelligent) are frequently judged to be more likely in a given individual, than the separate attributes making up the conjunction. Likewise given the presence of some hypothetical disease, experienced physicians judged conjunctions of different symptoms to be more likely than the individual symptoms (Tversky \& Kahneman, 1983).

Considered to be one of the most important probabilistic reasoning errors, a key focus of the present paper is the conjunction fallacy. The conjunction fallacy occurs when the probability of the conjunction of two events, $\mathrm{P}(\mathrm{A} \& \mathrm{~B})$, is judged to be more likely than the probability of one or both of the corresponding component events $\mathrm{P}(\mathrm{A})$ and $\mathrm{P}(\mathrm{B})$, an outcome which violates the conjunction rule in probability theory. Nobel prize winning psychologist and decision theorist, Daniel Kahneman, has described the extension rule and the conjunction rule which derives from it, as "the simplest and most fundamental qualitative law of probability" (Tversky \& Kahneman, 1983, p. 294). The fact that it is routinely violated in everyday judgements is therefore of fundamental importance, theoretically and practically.

The conjunction rule may be expressed as follows:

$\mathrm{P}(\mathrm{A} \& \mathrm{~B})=\mathrm{P}(\mathrm{A}) \times \mathrm{P}(\mathrm{B} \mid \mathrm{A})$

or equivalently

$\mathrm{P}(\mathrm{A} \& \mathrm{~B})=\mathrm{P}(\mathrm{B}) \times \mathrm{P}(\mathrm{A} \mid \mathrm{B})$

Equations 1 and 2 state that the probability of the conjunction of two events, $\mathrm{A}$ and $\mathrm{B}$, is equal to the probability of one of the events, e.g., $\mathrm{P}(\mathrm{A})$, multiplied by the conditional probability of the other event, $\mathrm{P}(\mathrm{B} \mid \mathrm{A})$. For example, the probability that I will get a promotion and a company car is equal to the probability that I will get a promotion multiplied by the probability that I will get a company car given that $\mathrm{I}$ have got a promotion [i.e., $\mathrm{P}($ promotion and company car $)=\mathrm{P}($ promotion $) \times \mathrm{P}($ company car | promotion)]. Since probabilities (e.g., in this instance $\mathrm{P}(\mathrm{B} \mid \mathrm{A})$ ) are, by definition, less than or equal to unity it follows that:

$$
\mathrm{P}(\mathrm{A} \& \mathrm{~B})=\mathrm{P}(\mathrm{A}) \times \mathrm{P}(\mathrm{B} \mid \mathrm{A}) \leq \mathrm{P}(\mathrm{A})
$$

and therefore judgements such that:

$$
\mathrm{P}(\mathrm{A} \& \mathrm{~B})>\mathrm{P}(\mathrm{A})
$$

and/or

$$
\mathrm{P}(\mathrm{A} \& \mathrm{~B})>\mathrm{P}(\mathrm{B})
$$

violate the conjunction rule and such judgements are considered examples of the conjunction fallacy. 
Perhaps the best-known example of the conjunction fallacy is Tversky and Kahneman's (1983) vignette concerning a hypothetical person named Linda:

'Linda is 31 years old, single, outspoken and very bright, majored in philosophy and, as a student was deeply concerned with issues of discrimination and social justice and participated in anti-nuclear demonstrations' (Tversky \& Kahneman, 1983, page 297)

Having read this description, individuals are asked to rank various statements in order of their likelihood. These statements can either be consistent with the description (e.g., Linda is a feminist) or inconsistent with it (e.g., Linda is a bank teller). In addition, conjunctive statements which often contain both consistent and inconsistent statements (e.g., Linda is a bank teller AND is a feminist), are also included. In completing this task, the vast majority of individuals ranked the conjunction (Linda is a bank teller AND is a feminist) more likely than its component (Linda is a bank teller) thereby succumbing to the conjunction fallacy.

Another fundamental rule in probability theory is the addition (or disjunction) rule which states that:

$$
\mathrm{P}(\mathrm{A} \text { OR } \mathrm{B})=\mathrm{P}(\mathrm{A})+\mathrm{P}(\mathrm{B})-\mathrm{P}(\mathrm{A} \text { and } \mathrm{B})
$$

that is the probability of the disjunction of two events is equal to the sum of the probabilities of those two events minus the corresponding conjunctive probability. From equation 3 we know that $\mathrm{P}(\mathrm{A}$ and B) $\leq \mathrm{P}(\mathrm{A})$ and therefore $\mathrm{P}(\mathrm{A})-\mathrm{P}(\mathrm{A} \& \mathrm{~B}) \geq 0$. This implies that $\mathrm{P}(\mathrm{A}$ or $\mathrm{B}) \geq \mathrm{P}(\mathrm{B})$ and by extension $\mathrm{P}$ (A or $\mathrm{B}) \geq \mathrm{P}(\mathrm{A})$. Thus, judgements such that:

$$
\mathrm{P}(\mathrm{A} \text { OR } \mathrm{B})<\mathrm{P}(\mathrm{A})
$$

and/or

$$
\mathrm{P}(\mathrm{A} \text { OR B })<\mathrm{P}(\mathrm{B})
$$

violate the disjunction rule and such judgements are considered examples of the disjunction fallacy. Equation 6 represents the standard inclusive version of the disjunction rule. Here, either event A or event $\mathrm{B}$ or both events together are permitted to occur. Equation 9 below describes the exclusive version of the disjunction rule where either event A or event $B$ are permitted to occur but both events are not allowed to occur together:

$$
\mathrm{P}(\mathrm{A} \text { XOR } \mathrm{B})=\mathrm{P}(\mathrm{A})+\mathrm{P}(\mathrm{B})-2 \times \mathrm{P}(\mathrm{A} \text { and } \mathrm{B})
$$

For example, we might consider the likelihood that 'Linda is a feminist or Linda is an anarchist' while excluding the set of possibilities possibilities where she is both a feminist and an anarchist. The disjunction fallacy cannot be defined for exclusive disjunctions since in these cases it is perfectly possible for the exclusive disjunctive probability to be less than one or both component probabilities. Relative to the conjunction fallacy, violations of the disjunction rule have been relatively neglected in the research literature (Cf. Bar-Hillel \& Neter, 1993; Carlson \& Yates, 1989; Costello \& Watts, 2016; 2018; Nilsson, Winman, Juslin, \& Hansson, 2009). The present paper seeks to evaluate the processes underpinning conjunctive and disjunctive judgements within a unified framework and in the process, 
we develop our own model of joint probability judgement. First, we review some of the previous literature focussing on the more prominent research findings.

As Tentori, Crupi and Russo (2013) have pointed out, most existing accounts of the conjunction fallacy emphasise the role of the more likely component (which they refer to as the added conjunct) in accounting for the conjunction fallacy. For example, Tversky and Kahneman (1983) demonstrated that when the background evidence (e.g., Linda's description) was representative of an event (Linda being a feminist) the conjunction fallacy was more likely to occur than when the evidence was not representative of an event (Linda being a homemaker). That is to say, the fallacy was more likely to occur with the conjunctive event 'bank teller and feminist' than with the conjunctive event 'bank teller and homemaker'. Perhaps unsurprisingly this resulted in an emphasis being placed on the characteristics of the more representative event (e.g., feminist) which was also almost invariably the more likely event.

In other early research, Yates \& Carlson's (1986) signed summation account of the conjunction fallacy relied upon the concept of a qualitative likelihood index (QLI). It was proposed that likely events were subjectively assigned a positive value on the index, unlikely events a negative value and that probabilities were somehow derived from these values. The QLI value of the conjunction of a likely and an unlikely event was then assumed to be the arithmetic sum of these two numbers, which invariably was greater than the negative QLI for the unlikely event, hence giving rise to the fallacy. Signed summation offers an explanation as to why it is that the fallacy is rare with two unlikely events and why double fallacies sometimes occur with conjunctions of two likely events.

More generally, it had been widely believed that the fallacy was most likely to occur when a likely event was paired with an unlikely one (e.g., Thuring \& Jungermann, 1990). More recently Tentori et al. (2013) questioned whether this was always the case. They introduced the concept of inductive confirmation. Specifically, when an event (e.g., Linda being a feminist) was inductively confirmed by the background evidence (e.g., Linda's description) then the fallacy was more likely to occur than when the event (e.g., Linda owning an umbrella) was not confirmed by the evidence. Thus, for example, the fallacy was more likely to occur with the conjunctive event 'bank teller and feminist' than with the conjunctive event 'bank teller and umbrella owner' even though the probability of Linda owning an umbrella was probably greater than that of her being a feminist.

However, what these and other accounts of the fallacy fail to do is to directly examine the how different degrees of representativeness, inductive confirmation, or QLI values, combine to determine the actual probability assigned to the conjunctive probability. Their primary focus was on the presence or absence of the fallacy. Indeed, it is not clear how the degree of representativeness, the QLI value or degree of confirmation associated with individual component events is integrated in the determination of the corresponding conjunctive values. This has never been systematically investigated. Indeed, Gavanski and Roskos-Ewoldsen (1991) demonstrated that while single event probabilities might be conceivably based on degrees of representativeness, the conjunctive event 
probability did not seem to be so. While more recent theoretical perspectives including those of Costello and Watts, $(2014 ; 2016)$ and Nilsson et al. (2009) share our focus on the relationship between the component events and the corresponding joint events, in our case, we have directly examined this relationship using statistical means including regression analysis, within participants ANOVA and more recently multi-level linear modelling. Thus, by way of summary, in much of the existing literature, the focus on the more likely event was essentially conceptual and qualitative (utilising concepts such as representativeness or signed summation and more recently, inductive confirmation) rather than being quantitatively based. Our own approach has been substantively different. We have focussed on the relationship between the actual estimated component probabilities and the conjunctive probability estimate demonstrating that that for the most part, for conjunctive judgements only the less likely component has a direct influence on the outcome while for disjunctive judgements, this role is performed by the more likely component. In each case, for most judgements, the other component appears to play no direct role (anonymised references). Clearly our own results are difficult to reconcile with much of the existing literature.

How is it that individuals come to focus on only one component in making their judgements of the probability of joint events? First, individuals are able to form a view as to the likelihood of an event. From the perspective of subjective probability, this will be based on their prior experiences, as well as their beliefs and feelings. However, memory for past events is at least in part a construction rather than a faithful representation of what actually occurred (e.g., Carpenter \& Schacter, 2017; Devitt, Monk-Fromont, Schacter, \& Addis, 2016; Hyman, \& Loftus, 1998) and estimates of the frequency of past and possible future events are known to be subject to systematic distortion (Armantier, 2006; Combs \& Slovic, 1979, Hakes \& Viscusi, 2004; Lichtenstein, Slovic, Fischhoff, Layman, \& Combs, 1978; Odegard \& Lampinen, 2004). Nonetheless, whether from the subjectivist perspective or other conceptualisations of probability, judgements of joint probability are constrained by the rules of probability theory. However, evidence suggests that the majority of individuals are unaware of these rules (e.g., Yates \& Carlson, 1986).

Despite this lack of objective awareness, since uncertainty is a prominent aspect of everyday life strategies are needed to deal with it. We continuously confront events, including joint events, which are probabilistic in nature, some relatively trivial, e.g., the likelihood that it will be dry for the remainder of the week and into the weekend; that the bus will be late and traffic will be heavy; and some of greater importance, e.g., that mortgage rates will remain low and employment prospects will remain buoyant; that I manage to get an interview and prove to be the best candidate; that at least one or other of my financial advisors will be able to get the deal that I need. As part of our everyday experience we encounter events such as these all the time and crucially as the outcomes become known to us we gain some awareness of the relationship between the component probabilities and their conjunctions and disjunctions, albeit perhaps an imprecise awareness. It is likely that some of 
these relationships are first encountered in childhood and perhaps a simple, if somewhat trivial, example might serve to illustrate this:

Colin, Denis and Peter all play soccer together after school in Colin's garden. Colin acts as referee and Peter and Denis play each other as opposing teams. Denis has lots of other commitments and only makes it to the game $20 \%$ of the time while Peter is almost always there, around $85 \%$ of the time. Colin comes to realise that the chances of having a game are more determined by the likelihood that Denis is able to attend. Utilising this insight, Colin comes to realise that the conjunctive event of both Denis and Peter being present is most heavily dependent on the likelihood of Denis attending. In this sense, he comes to realise that the likelihood of a conjunctive event (e.g., Denis and Peter attending) is mostly determined by the likelihood of the less likely event (e.g., Denis attending).

Peter's father is at home after school $75 \%$ of the time to take him to Colin's to play football. Peter's mother is at home less often, around $35 \%$ of the time, and on these occasions sometimes she takes him. Peter comes to realise that his chances of playing football are mostly dependent on whether his father is at home. So in evaluating the disjunctive event of either his father or his mother taking him to Colin's, Peter focusses on whether or not his father is likely to be at home. In this sense he comes to realise that the likelihood of a disjunctive event (e.g., his dad or his mum taking him to Colin's) is mostly determined by the likelihood of the more likely event (e.g., his dad taking him to Colin's).

Beyond these trivial examples, objectively, the laws of probability ensure that it is the less (more) likely event that accounts for most of the variance in the conjunctive (disjunctive) outcome ${ }^{\mathrm{i}}$. Thus, without the need to actually work out the probabilities, experience allows us, through a process of associative learning and perhaps without conscious awareness, to focus on the less likely event in the conjunctive case and the more likely in the disjunctive since these have the much greater impact on the respective joint probability outcomes. Over the years we have accumulated evidence consistent with this possibility (e.g., Anonymised 1-5) as have other researchers (e.g., Kariyazono, 1991; Lu, 2016; Thuring \& Jungermann, 1990; Wyer, 1970). However, we have yet to consider conjunctive and disjunctive judgements containing the same component events. Doing so will allow us to test the predictions of our model regarding the role of the component events in determining the joint event probabilities. More importantly this will allow us to explore the normatively crucial relationship between the conjunctive-less likely difference and the more likely-disjunctive difference. As we will show below these two differences form the essential ingredients of the addition rule in probability theory and have been neglected or remain unexplained in the existing literature.

We now outline the key assumptions and predictions that stem from our theory.

1. In subjective probability judgement, the process through which joint probabilities are estimated initially utilises a reference point or anchor. In the case of the conjunctive (disjunctive) probability this is usually, but not invariably, the less (more) likely component event probability. On relatively rare occasions these respective roles may be reversed. Having selected the reference point, we further propose that some decision rule is then applied. Importantly, we argue that the application of this decision rule is essentially qualitative in nature resulting in a quasi-random adjustment from the reference point, either 
positive or negative in direction. An implication of this is that the smaller (larger) component probability will account for all, or the majority, of the variance in the conjunctive (disjunctive) event probability. This assertion will be tested utilising regression and linear multilevel modelling analyses.

2. For those typical responses where the less (more) likely component serves as the reference point for the conjunctive (disjunctive) judgement, while the adjustment from the reference point is quasi-random, it will potentially be constrained by the position of the reference point relative to the end points of the probability continuum: zero and unity ${ }^{\text {ii }}$. For example, for those avoiding the fallacy, in the conjunctive (disjunctive) case, the magnitude of the negative (positive) adjustment with respect to the reference point will potentially be constrained by the space between the reference point, i.e., the smaller (larger) component probability and the zero (unity) point on the probability continuum. In this sense, although quasi random, the magnitude of the adjustment will be influenced by the magnitude of the reference point probability. In essence, these quasi-random adjustments are equal to the conjunctive-less likely probability difference and the more likely-disjunctive probability difference. Thus, with the exception of those rare cases where it can be demonstrated that the atypical component serves as the reference point for the joint probability judgement, it follows that only the less (more) likely component probability should be statistically significant in determining the conjunctive-less likely (more likely-disjunctive) difference. It is important to note that this is contrary to standard probability theory where both components would be expected to play an equal part in determining the component-joint event differences. These propositions will be tested using linear multilevel modelling analyses.

3. According to standard probability theory, it follows from the addition rule that the conjunctive-less likely difference should be exactly equal to the more likely -disjunctive difference. Rearranging equation 6 :

$$
\mathrm{P}(\mathrm{A} \text { and } \mathrm{B})-\mathrm{P}(\mathrm{B})=\mathrm{P}(\mathrm{A})-\mathrm{P}(\mathrm{A} \text { OR } \mathrm{B})
$$

However, according to our account, the conjunctive-less likely difference is unrelated to the more likely-disjunctive difference. This is because the above-mentioned differences (although potentially bounded by the component events; see point 3 ) are quasi random in nature and thus are unlikely to be correlated.

In what follows, we present two studies in which the above expectations are tested.

\section{STUDY 1}

Study 1 utilises scenarios in which background information is provided concerning fictitious individuals followed by a number of statements (single event and joint event) some consistent with the background information, others inconsistent or else conflicted. To enhance comparability with 
previous research findings, the scenarios we use are based on those from the existing research literature including Linda, Bill, and Health Survey (Tversky \& Kahneman, 1983) as well as Ollie (Tentori et al. 2013). Disjunctions were included in each scenario (replacing 'and' in the conjunction by 'or'). They were either inclusive where both components were permitted to occur or exclusive (XOR) where only one or other was permitted to occur to the exclusion of the other.

\section{Method}

\section{Participants.}

An opportunity sample consisting of eighty participants (34 females) took part in the study. Participants, who were students and staff from a University in the North West of England, ranged in age from 18-50 (average 23.05; SD 5.98). They were randomly assigned to the inclusive or exclusive disjunction condition (40 in each group). All participants produced data allowing estimates of the incidence of conjunction fallacy to be derived. As we note above, since the XOR exclusive disjunctions can give rise to probabilities which are less than one or both components without violating the rules of probability, disjunction fallacy estimates were derived from the first group only.

\section{Materials}

Participants completed five scenarios. Single event probabilities $\mathrm{P}(\mathrm{A})$ and $\mathrm{P}(\mathrm{B})$ were as follows: Linda (feminist, bank teller), Bill (accountant, plays jazz), Ollie (music lessons, mountaineer), Health Survey, referred to as Tom (over 55, heart attack), and Rick (athletic, under 25). After reading some brief information concerning the protagonist featured in each vignette, participants judged the likelihood of two single event statements and their conjunction. The order of statements within each vignette was randomised. Participants responded on a 0-100 point scale. Prior to completing the task, participants received instructions on the use of the scale. The Ollie vignette is reproduced below. Copies of all vignettes are available from the corresponding author

\section{Ollie has a degree in violin performance.}

Now please judge how probable each of the following statements is by entering a number between 0 and 100 for each one:

\begin{tabular}{|l|l|}
\hline Statement & $\begin{array}{l}\text { Enter a number } \\
\text { between 0 and 100 }\end{array}$ \\
\hline Ollie gives music lessons & \\
\hline Ollie is an expert mountaineer & \\
\hline Ollie is an expert mountaineer and gives music lessons & \\
\hline
\end{tabular}

Participants then completed an inductive confirmation task which is the subject of another study. After this, they then estimated the value of the disjunction probability. For this purpose, participants were randomly assigned to the inclusive or the exclusive disjunction condition:

Inclusive:

Ollie has a degree in violin performance. 
Now please judge how probable the following statement is by entering a number between 0 and 100:

\begin{tabular}{|l|l|}
\hline Statement & $\begin{array}{l}\text { Enter a number } \\
\text { between 0 and 100 }\end{array}$ \\
\hline Ollie is an expert mountaineer OR gives music lessons OR both. & \\
\hline
\end{tabular}

Exclusive:

Ollie has a degree in violin performance.

Now please judge how probable the following statement is by entering a number between 0 and 100:

\begin{tabular}{|l|l|}
\hline Statement & $\begin{array}{l}\text { Enter a number } \\
\text { between 0 and 100 }\end{array}$ \\
\hline $\begin{array}{l}\text { Ollie is an expert mountaineer OR gives music lessons BUT } \\
\text { NOT both. }\end{array}$ & \\
\hline
\end{tabular}

\section{Procedure.}

Participants were briefed as to the nature of the study and verbal consent was obtained. They completed the questionnaire in their own time, in the presence of the experimenter. At the end of the session they received a written debrief. The study was administered in accordance with the ethical guidelines of the British Psychological Society and the University of Central Lancashire Ethics Committee.

\section{Design/Statistics}

The number of fallacies summed over the Linda, Bill, Rick and Tom scenarios (the DV) was compared with the presence or absence of the fallacy in the Ollie scenario as the IV. Separate analyses were conducted for the conjunctions and inclusive disjunctions. The Ollie scenario was selected as the basis for the IV because this was where the relative incidence of the fallacy was closest to $50 \%$.

The relationship between the joint event probability as DV (separate analyses were conducted for the conjunctive and disjunctive probabilities) and the more likely and less likely component probabilities as IVs was evaluated using multilevel modelling (MLM). At the lowest level of the analysis were the repeated measures across the five scenarios. Individual participants constituted the next level with the above-mentioned IVs as predictors. A second set of MLM analyses were conducted with identical DV and IVs but excluding those judgements where the conjunctive (disjunctive) probability was set exactly equal to the less (more) likely component. This was done to confirm the role of the IVs in those judgements where there was a positive or negative difference between the joint event and the component probabilities.

The conjunctive-less likely probability difference and the more likely-disjunctive probability difference were analysed as separate DVs utilising MLM with the same structure as in the preceding analysis. IVs were the more and less likely component probabilities. The presence or absence of the 
fallacy was included as an additional predictor (constituting an additional level in the MLM). We also included two interaction terms: the interaction of the fallacy variable with the more and less likely component probabilities, respectively. These interaction terms are potentially important since where they are statistically significant, it indicates that the role of the less and more likely component probabilities (in determining the joint event - component probability differences) varies significantly between those committing the fallacy and those avoiding it.

For MLM analyses we attempted to model the repeated measures component (reflecting the extent to which participants' estimates across scenarios may violate the independence assumption) by fitting various variance-covariance structures, including heterogeneous variance models, the standard 'independence' model (ID, identical variance, zero covariances) as well as other models with homogeneity of variance assumed. The -2 Log Likelihood statistic was used to select the best performing model. Differences in this statistic were evaluated utilising Chi squared. In all the MLM analyses, where possible we modelled the random intercept and slope parameters using the UN model.

In all MLM analyses, in both Studies, we report only the outcomes for the best fitting models for which convergence was achieved. Relevant inferential statistics (AICC values, auto-regressive or ante dependence rho values and random effects parameters) and significance levels for all models are available from the corresponding author as well as outcomes for the random intercept and slope parameters.

\section{Results}

\section{Incidence of the Fallacy.}

On average, each participant committed 1.86 (SD 1.59) conjunction fallacies out of a maximum of 5. Among those participants receiving the inclusive disjunction problem, the average number of disjunction fallacies was 1.78 (SD 1.62) out of a maximum of 5. In order to evaluate whether or not the tendency to commit or avoid the fallacy was consistent across a number of judgements, we focussed on the outcome of the Ollie scenario where the incidence of the fallacy was closest to $50 \%$, i.e., $44 \%$ in the conjunctive case and 53\% in the disjunctive. This allowed us to divide the sample into approximately equal groups. We examined the incidence of the conjunction fallacy in the remaining scenarios, comparing those who committed the conjunction fallacy in the Ollie scenario with those avoiding it. The average for the former group was 2.11 (SD 1.02) and for the latter group 0.89 (SD 1.23). The difference was statistically significant, $\mathrm{t}(78)=-4.78, \mathrm{p}<.001$. In the disjunctive case, the average in the remaining scenarios for the 'Ollie fallacy' group was 2.10 (SD 1.04) while for the 'Ollie no fallacy' group it was 0.32 (SD 0.48). The difference was again statistically significant, $\mathrm{t}(28.62)=7.04, \mathrm{p}<.001$. Thus, it seems clear that those committing the fallacy in the Ollie scenario were significantly more likely to commit it in the remaining scenarios. In the analyses that follow, 
two new variables were created for each scenario: $\mathrm{P}($ less likely) was defined as the smaller of $\mathrm{P}(\mathrm{A})$ and $\mathrm{P}(\mathrm{B})$ and $\mathrm{P}($ more likely) defined as the larger of $\mathrm{P}(\mathrm{A})$ and $\mathrm{P}(\mathrm{B})$.

\section{Modal Responses}

As to the actual conjunctive probability itself, examination of the individual responses revealed that in 166 cases ( $42 \%$ of the total) the conjunction was assigned a probability exactly equal to the less likely component. However, excluding those cases where $\mathrm{P}($ less likely $)=\mathrm{P}($ more likely $)=\mathrm{P}($ Conjunction) (in these cases for the most part all events were assigned probabilities of $50 \%$ ), 76 judgements (19\% of the total) were such that the conjunctive probability was assigned the same value as the less likely component. For the inclusive disjunctions, $40(20 \%)$ responses were such that the disjunctive probability was assigned the same value as the more likely component. However, again excluding those cases where $\mathrm{P}($ less likely $)=\mathrm{P}($ more likely $)=\mathrm{P}($ Disjunction $), 17$ judgements $(9 \%$ of the total) were such that the disjunctive probability was assigned the same value as the more likely component. These tendencies are consistent with our first proposition that the component event probabilities serve as reference points for the joint event judgements. However, again excluding those cases where $\mathrm{P}($ less likely $)=\mathrm{P}($ more likely $)=\mathrm{P}($ Joint Event $)$, there were 10 cases $(3 \%$ of the total) where the conjunctive probability was set exactly equal to the more likely event probability and 14 cases (7\% of the total) where the inclusive disjunctive probability was set exactly equal to the less likely event probability. Thus, it appears that a minority of participants may use the other component as a reference point when making their joint event probability judgements.

\section{The Role of the More Likely and Less Likely Probabilities in Determining the}

\section{Conjunctive and Disjunctive Probabilities}

Despite the small number of participants who may behave in a contrary fashion, our model predicts that a majority of participants will use the less (more) likely component as the reference point for the conjunctive (disjunctive) probability judgement and that the other component probability will play no direct role. The implication of this is that the less (more) likely component should account for all or most of the statistically significant variance in the conjunctive (disjunctive) probability. To test this proposition, we conducted two sets of MLM analyses.

The results (parameter estimates) for the two best fitting models are set out in Table 1. Consistent with our first proposition, for the conjunctive event probability, only the less likely component was statistically significant in predicting the value of the conjunction. The opposite was true for the disjunctive judgements with only the more likely event a significant predictor.

<insert Table 1 about here>

Clearly, the fact that a substantial number of participants set the conjunctive (disjunctive) probability exactly equal to the less (more) likely probability contributed to the results we obtained. With the aim of controlling for this influence, we repeated the above two analyses (retaining the same 
repeated measures variance-covariance structures) in order to establish the relative importance of the component probabilities for those judgements where, respectively, the conjunctive and disjunctive probabilities were not set exactly equal to the less and more likely component probabilities. The results are set out in the third and fourth rows of Table 1. As was the case with the preceding two analyses and consistent with our first proposition, in the conjunctive case it is the less likely component which alone accounts for significant variance whereas in the disjunctive case this role is performed by the more likely component. The means values for the more and less likely components and for conjunctive and disjunctive probabilities are set out in Table 2.

<insert Table 2 about here>

The Role of the More Likely and Less Likely Component Event Probabilities in determining the Conjunctive-Less Likely Probability Difference and the More LikelyDisjunctive Probability Difference: MLM Analyses.

We investigated the extent to which the conjunctive-less likely difference and the more likelydisjunctive difference are dependent on the two component probabilities, in particular whether, as in the preceding analyses, influence is limited to the less likely component in the conjunctive case and the more likely component in the disjunctive. Thus, two MLM analyses were conducted with the conjunctive probability minus less likely component probability and the more likely component minus the disjunctive probability as DVs. IVs were the more and less likely component event probabilities and the absence or presence of the fallacy (coded respectively as 0 and 1). We also included the fallacy by less likely component and fallacy by more likely component event interaction terms as predictors.

For both the conjunctive-less likely component probability difference and the more likely component-disjunctive probability difference, the ID model produced the best fit. The parameter estimates for these two best fitting models are set out in in the final seven rows of Table 1. The row labelled Main Effect 1 relates to those individuals avoiding the fallacy; the row labelled Fallacy Interaction provides an indication of whether the influence of the predictors differs significantly between those committing the fallacy and those avoiding it; lastly the row labelled Main Effect 2 relates to those individuals committing the fallacy (here inferential statistics were obtained by rerunning the analysis reverse coding the zero-one fallacy variable). In relation to the conjunctive-less likely probability difference, the less likely component probability was statistically significant irrespective of whether or not the fallacy was committed. While the more likely component had no significant influence for those avoiding the fallacy, it did just reach significance for those committing the fallacy. (However, this outcome is somewhat ambiguous since the beta weight in question did not differ significantly from that for those avoiding the fallacy where it was not significant.) Regarding 
the difference between the more likely component probability and the disjunctive event probability, examination of the parameter estimates reveals that consistent with prediction, only the more likely component event probability exerted an influence, both for those participants committing the fallacy and for those avoiding it. For both the conjunctive-less likely and the more likely-disjunctive analyses, the absence of an interaction between the fallacy variable and the component event probabilities indicated that the strength of the relationship between the respective component event probabilities and the corresponding difference measures did not differ between those committing the fallacy and those avoiding it. With one minor qualification, proposition 2 is therefore supported.

For those committing the fallacy, it is noteworthy that the beta coefficient for the less likely component probability was negative, suggesting that as the magnitude of the less likely event increased so the difference between it and the conjunctive estimate decreased. The coefficient was also negative for those avoiding the fallacy. For disjunctive judgements, the beta coefficient on the more likely event was positive both for those committing the fallacy and for those avoiding it. Both these outcomes are consistent with proposition 2, namely that the quasi random adjustment is constrained by the interval between the relevant component probability (the reference point) and the end of the probability continuum (zero or one).

The Relationship between the Conjunctive-Less Likely and the More Likely-Disjunctive

\section{Probability Differences}

According to the addition rule in probability theory, the conjunctive-less likely difference is exactly equal to the more likely-disjunctive difference. Thus, even allowing for some degree of random error it would be expected that these two quantities should not differ significantly and should be significantly correlated. According to our model, since these two quantities are essentially quasirandom adjustments from the relevant component probability reference points, there is no reason to expect that they will be correlated, nor is it necessarily the case that the two differences should be of similar magnitude. Inspection of Table 3 reveals that consistent with our model, the more likelydisjunctive and the conjunctive-less likely differences are not significantly correlated in any of the scenarios. Furthermore, in two of the scenarios, the two quantities differ significantly.

$<<$ Insert Table 3 about here>>

To supplement the bivariate and univariate results, we ran a series of MLM analyses with the conjunctive-less likely difference as DV and the more likely-disjunctive difference as IV. Consistent with the results set out in Table 3, the average slope parameter, .042, was not statistically significant, $\mathrm{t}=0.64, \mathrm{p}=.529$. Thus, proposition 3 is supported.

\section{Discussion}

It is worthy of note that the modal response was to set the conjunctive (disjunctive) probability exactly equal to the less (more) likely component probability. However, contrary to the 
overall trend, a small number of participants appear to have set the joint probability exactly equal to the other component (i.e., conjunctive set equal to the more likely and disjunctive to the less likely component probability). MLM modelling revealed that, consistent with our prediction, only the less (more) likely component was statistically significant in predicting the conjunctive (disjunctive) probability. Furthermore, the same result was obtained even after we excluded those judgements such that the joint event was set exactly equal to the relevant component.

It follows from probability theory that the conjunctive-less likely difference and the more likely-disjunctive difference should be exactly equal and that both component probabilities should play an equal role in their determination. However, according to our model there is no reason to suppose that the two quantities are related and our results largely confirm this. First, only the more likely component plays a statistically significant role in determining the more likely-disjunctive difference irrespective of whether or not the fallacy was committed. Second, in the analysis of the conjunctive- less likely difference, for those avoiding the fallacy, only the less likely component played a statistically significant role. However, for those participants committing the fallacy, although the less likely event accounted for most of the variance, it was also the case that the more likely component was statistically significant. A possible explanation for this is that a minority of participants may have used the more likely component as the reference point rather than the less likely event. Indeed, it was observed that a small number of participants set the conjunctive event exactly equal to the more likely component. Such judgements will give rise to the conjunction fallacy.

Contrary to probability theory, we contend that the conjunctive-less likely difference and the more likely-disjunctive difference will be unrelated. Our results confirm this. The two differences were not significantly correlated in any of the five scenarios and in two of the five, their magnitudes differed significantly. These results were further borne out by the MLM analyses.

\section{STUDY 2}

Study 1 utilised the type of scenarios that have featured prominently in the existing research literature. However, few studies have examined regular, naturally occurring real world events where the participant might possess some knowledge of the actual frequencies of the events in question. An exception is Costello and Watts' (2014) study in which participants were asked to provide estimates of the likelihood of weather events or combinations of weather events. It would be of interest to establish whether our expectations, as set out above, would be manifested in these types of judgements. This is the focus of our second study. We used exactly same weather event combinations as were used by Costello and Watts (2014).

In a further methodological variation from Study 1, we asked half of our participants to provide their responses in terms of probabilities while the other half provided them in terms of frequencies. By way of an example, the statement: 'What is the probability that it will rain today?' 
requires a judgement of probability while the statement: 'Imagine 100 days selected at random, on how many of these would rain occur?' elicits a judgement of frequency. Previous research has shown that when individuals respond in terms of frequencies, the incidence of the conjunction fallacy is greatly reduced, (e.g., Fiedler, 1988). Therefore, the possibility exists that frequency judgements may be such that the relationship between the components and the joint events may be more in line with probability theory than is the case with probability-based judgements. However, as with Study 1, our primary aim is to test the propositions underpinning our model as set out above.

\section{Method}

Participants. Sixty participants (34 females) with an average age of 21.20 years (SD 2.21; range 18-27) took part in the study. They were students from a University in the North West of England. Participants were randomly assigned, half to the frequency and half to the probability condition.

Materials and Procedure: The study was administered in accordance with the ethical guidelines of the British Psychological Society and the University of Central Lancashire Ethics Committee. As already stated, materials were closely based on those used by Costello and Watts (2014; Experiment 1). Specifically, we gathered estimates from 60 participants for 12 pairs of weather events. We used the same two sets of weather events (the set cloudy, windy, sunny, thundery and the set cold, frosty, sleety) as Costello and Watts (2014) to form the conjunctive and disjunctive joint event pairs. These pairs of weather events were formed by pairing each member of the first set with every member of the second set, placing and/or between the components as required. Examples of joint events were cloudy and cold, cloudy and frosty, as well as windy or frosty, windy or cold, etc. Participants provided estimates for the joint events and the individual component events. One group of participants $(N=30)$ answered the questions in the probability format:

What is the probability that the weather will be $X$ on a randomly selected day in North West England?

A second group $(N=30)$ answered the questions in in the frequency format:

Imagine a set of 100 different days, selected at random. On how many of those 100 days do you think the weather in North West England would be $X$ ?

In each case, $\mathrm{X}$ could be a single event such as cloudy, a conjunctive event such as cloudy and cold, or a disjunctive event such as cloudy or cold. Thus, each participant produced twelve conjunctive and twelve disjunctive estimates together with estimates for the 7 component events (either probabilities or frequencies); meaning that each participant produced 31 estimated probabilities/frequencies. 
Design/Statistics. With one or two additions, we utilised the same design and analytical approach as in Study 1. To establish whether the propensity to commit the fallacy in one scenario increased the likelihood of committing it in other scenarios, we used bivariate correlation to compare the incidence of the fallacy in half of the weather event combinations with that in the remaining half.

The present study also included a series of regression analyses, one for each participant. These were repeated with different DVs including the conjunctive probability and the disjunctive probability. In both cases, IVs were the more and less likely component probabilities.

Lastly, all MLM analyses were repeated with the frequency-probability response format as an additional dichotomous IV. To establish whether the impact of the other predictors, e.g., the component probabilities, differed according to whether responses were made in the frequency or probability format, we included the interaction of the response format dichotomous IV with each of the other predictors as additional IVs.

\section{Results}

\section{Incidence of the Fallacy}

Across participants, disjunction and conjunction fallacies averaged 7.52 (SD 3.07) and 6.12 (SD 2.58) respectively out of a maximum of 12. Thus, in total participants committed 13.63 (SD 4.68) fallacies on average out of a maximum of 24 . The incidence of conjunction fallacies did not differ between the frequency and probability conditions, $\mathrm{t}=0.81, \mathrm{p}>.05$. However, there were significantly more disjunction fallacies in the frequency condition, $8.43, \mathrm{SD}=2.61$, compared to the probability condition, 6.60, $\mathrm{SD}=327, \mathrm{t}=2.40, \mathrm{p}=.019$.

In order to determine whether or not the tendency to commit or avoid the fallacy was consistent across a number of judgements, we calculated the total number of fallacies summed over half of the joint weather events set out in Table 4 and correlated this sum with the total number of fallacies summed over the remaining half of the joint weather events, doing this separately for conjunction and disjunction fallacies. The number of conjunction fallacies for the first six joint weather events was significantly correlated with the number of fallacies for the remaining six, $r=.327$, $\mathrm{p}=.011$. The equivalent figures relating to the disjunction fallacy were $\mathrm{r}=.503 ; \mathrm{p}<.001, \mathrm{~N}=60$ in both cases. Thus, as was the case in Study 1, those who tended to avoid or commit the fallacy in one judgement were more likely to do so in other judgements.

$<<$ Insert Table 4 about here >

\section{The Role of the More Likely and Less Likely Probabilities in determining the}

\section{Conjunctive and Disjunctive Probabilities: Individual Responses and MLM Analyses}

In considering the relative influence of the component event probabilities in determining the probabilities assigned to the joint events, it is worthy of note that $11 \%(8 \%)$ of responses were such that the conjunctive (disjunctive) probability was set exactly equal to the less (more) likely component 
probability. In both cases, these were the modal responses. However, some participants exhibited the opposite pattern. Specifically, for $6 \%$ of responses the conjunctive event was set exactly equal to the more likely component. The same proportion of responses, $6 \%$, were such that the disjunctive event was set exactly equal to the less likely component.

We further explored the role of the component events in determining the joint probability by conducting two sets of MLM analyses. For each set, the joint event probability (conjunctive or disjunctive) was the dependent variable and the component event probabilities served as IVs.

Parameter estimates from the two best fitting models make up the first two rows of Table 5. While the less likely event was significant as a predictor of the conjunctive probability and the more likely as a predictor of the disjunctive probability, interestingly the more likely component was also statistically significant as a predictor of the conjunctive event and the less likely was also a significant predictor of the disjunctive event. So as to test our proposition concerning the relative influence of each component in predicting the joint event probability, we made use of Xu's (2003) procedure. Xu's formula allows us to calculate the partial coefficient representing the unique variance associated with a specific predictor, $\mathrm{X}$. In the expression below, $\mathrm{v} 2$ is the variance in the residuals associated with the full model and $\mathrm{v} 1$ the variance in the residuals for the model excluding predictor $\mathrm{X}$.

$$
\Omega_{\mathrm{x}}{ }^{2}=1-\mathrm{v} 2 / \mathrm{v} 1
$$

Making use of this formula, in predicting the conjunctive event, the variance associated with the less likely event was $40.52 \%$ while that associated with the more likely event was $5.92 \%$. For the disjunctive estimate the variance associated with the more likely event was $38.42 \%$ while that associated with the less likely event was $16.40 \%$. So although both events were significant predictors, consistent with proposition 1, the less likely event accounts for most variance in the conjunctive probability while the more likely event fulfils this role in the case of the disjunctive probability. So as to contextualise these percentages, we conducted three analyses with simulated data constrained to be consistent with the normative model (i.e., consistent with equations 1 and 6). These demonstrated that the less likely component uniquely accounted for between $54.8 \%$ and $58.4 \%$ of the total variance in the conjunctive probability while the more likely event accounted for between $1.4 \%$ and $2.0 \%$. In the disjunctive case, the more likely event uniquely accounted for between $54.3 \%$ and $59.1 \%$ of the variance, the less likely event between $1.5 \%$ to $2.0 \%$.

$$
<<\text { Insert Table } 5 \text { about here >> }
$$

\section{The Role of the More Likely and Less Likely Probabilities in Determining the}

\section{Conjunctive and Disjunctive Probabilities: Regressions for Each Participant}

While both component probabilities were statistically significant in the results set out in the preceding section, nonetheless this outcome remains consistent the proposition that participants select just one or other of the component probabilities as the reference point for their joint probability judgement. While our results suggest that most appear to select the less likely for the conjunctive 
estimate and the more likely for the disjunctive, a minority appear to focus solely on the other component (more likely for the conjunctive, less likely for the disjunctive). However, another possibility, more consistent with probability theory, is that most participants in fact utilise both components in making their judgements.

In order to explore these two possibilities, we ran separate regressions for each participant with the 12 joint probability estimates as the dependent variable and the component probabilities as predictors. In the conjunctive case, only the less likely component was statistically significant, $\mathrm{p}<.05$, in 22 cases, average squared semi partial correlation coefficient, $._{\mathrm{sp}}{ }^{2}=482$. In view of the limited power, with $.10>p>.05$, in a further 5 cases, again only the less likely component was statistically significant, average $\mathrm{r}_{\mathrm{sp}}{ }^{2}=.216$. Against the trend, in 3 cases only the more likely component was statistically significant, $\mathrm{p}<.10$. For these cases, average $\mathrm{r}_{\mathrm{sp}}{ }^{2}=.251$. Only 5 participants produced estimates for which both components were statistically significant, $\mathrm{p}<.10$. Here, for the less and more likely components, average $\mathrm{r}_{\mathrm{sp}}{ }^{2}$ was.403 and .105 respectively. Lastly, for the remaining 25 participants, neither component probability was statistically significant, $\mathrm{p}>.10$.

For the disjunctions as DV, the more likely component only, was statistically significant, $\mathrm{p}<.05$, in 8 cases, average $\mathrm{r}_{\mathrm{sp}}{ }^{2}=337$. With $.10>\mathrm{p}>.05$, in a further 3 cases, again only the more likely component was statistically significant, average $\mathrm{r}_{\mathrm{sp}}{ }^{2}=.265$. The less likely component also featured prominently and in 9 cases, only it was statistically significant in predicting the disjunctive estimate, $\mathrm{p}<.05$. For these cases, average $\mathrm{r}_{\mathrm{sp}}{ }^{2}=.422$. Furthermore, with $.10>\mathrm{p}>.05$, again only the less likely component was significant in a further three cases, average $\mathrm{r}_{\mathrm{sp}}{ }^{2}=.259$. Only 7 participants produced estimates for which both components were statistically significant, $\mathrm{p}<.10$. Here, for the more and less likely components, average $\mathrm{r}_{\mathrm{sp}}{ }^{2}$ was.244 and .201 respectively. For the remaining 30 participants, neither component probability was statistically significant, $\mathrm{p}>.10$.

To summarise, it was generally found that only one component was statistically significant as a predictor of the joint event with the beta weight for the other not significantly different from zero. In these cases, the less likely event was prominent as the sole predictor for the conjunctive probability while for the disjunctive probability both components featured (approximately) equally often as sole predictor. It is worthy of note that for half of the participants, neither component was statistically significant at $\mathrm{p}<.10$. This is perhaps not entirely surprising since we have proposed that the joint probabilities are in part the product of a quasi-random process which, in the context of the regression model would serve to increase the error variance, potentially masking the effect of variation in the reference point probability.

The Relationship between the Conjunctive-Less Likely and the More Likely-Disjunctive Probability Differences: Univariate, Bivariate and MLM Analyses

Our model predicts that for the majority of participants, the conjunctive-less likely and the more likely- disjunctive differences represent quasi-random adjustments from the less and more likely 
component probabilities respectively. Thus, according to our account, the two differences should be unrelated. By way of contrast, probability theory (the addition rule) requires that the conjunctive-less likely and the more likely- disjunctive difference should be exactly equal. Even allowing for some degree of error in individual judgements, these two quantities would not be expected to differ significantly and should be significantly correlated. In fact, inspection of Table 6 reveals that the two differences are significantly correlated in only 2 of the 12 joint weather events. Furthermore, for 5 of the joint events, the two measures differed significantly with the difference approaching significance in a further 2. On balance these results are consistent with proposition 3 .

$$
<<\text { Insert Table } 6 \text { about here >> }
$$

To augment the univariate and bivariate results, we utilised MLM analysis in order to test the relationship between the conjunctive-less likely and the more likely-disjunctive probability differences within a single model. The former was the dependent variable and the latter the predictor. Consistent with the bivariate results, the estimated average slope parameter, specifying the relationship between the two differences, did not differ significantly from zero, beta $=.008, \mathrm{t}=0.25$, $\mathrm{p}=.801$. Thus, consistent with proposition 3 , the conjunctive-less likely difference and the more likelydisjunctive differences shared little if any common variance.

\section{The Role of the More Likely and Less Likely Component Event Probabilities in} determining the Conjunctive-Less Likely Probability Difference and the More Likely-

\section{Disjunctive Probability Difference: MLM Analyses.}

To explore the roles played by the component probabilities in determining the conjunctiveless likely and the more likely-disjunctive probability differences, we ran separate MLM analyses with the conjunctive-less likely and the more likely-disjunctive probability differences as DVs and with the component probabilities and the fallacy measure (present versus absent) as IVs. As in Study 1 , in order to establish whether or not the influence of the component probabilities on the joint eventcomponent event probability differences differed between those committing the fallacy and those avoiding it, we also included the interaction terms between the fallacy and the component probabilities as additional predictors.

Parameter estimates for the best fitting models are presented in bottom six rows of Table 5. Avoidance of the fallacy (see the rows labelled Main Effect ${ }_{1}$ ) is associated with the pattern of results that was predicted whereby only the less likely (more likely) event is statistically significant in predicting the conjunctive-less likely (more likely-disjunctive) difference. In contrast to those avoiding the fallacy, it is noteworthy that, for those committing it (the rows labelled Main Effect ${ }_{2}$ ), both component event probabilities significantly influence the probability assigned to the joint eventsingle event differences. These outcomes are also reflected in the relevant fallacy by component interaction terms, which are both statistically significant, reflecting the fact that the relative influence of the predictors is different for those committing the fallacy and those avoiding it. 


\section{Discussion}

As with Study 1, those who had a tendency to commit the fallacy in one scenario were more likely to do so in the other scenarios. It is noteworthy that the modal response was to set the conjunctive (disjunctive) probability exactly equal to the less (more) likely event. However, as was the case in Study 1, a small minority exhibited the opposite tendency setting the conjunctive (disjunctive) probability exactly equal to the more (less) likely event.

Unlike the outcome in Study 1, the MLM analyses revealed that both components were statistically significant in predicting the conjunctive and disjunctive probabilities. However, consistent with proposition 1, the less likely event accounted for most of the variance in the conjunctive probability, $41 \%$ compared with just $6 \%$ for the more likely probability. A similar, if somewhat less emphatic pattern emerged for the disjunctive event, with the more likely component and the less likely component accounting for $38 \%$ and $16 \%$ of the variance respectively. In the case of the reference point probability (less likely in the conjunctive case, more likely in the disjunctive) it is worthy of note that the above percentages are somewhat lower than the simulation for the normative model referred to above where the percentage averaged out at between $54.3 \%$ and $59.1 \%$. Conversely, we found that the other component probability accounted for more of the variance than was the case in the normative model where this component accounted for only $1.4 \%$ to $2 \%$. Since the Costello and Watts $(2014 ; 2106)$ model predicts that estimated probabilities are linear transformations of the actual objective probabilities ${ }^{\mathrm{iii}}$, a simulation revealed that their model yields similar percentages to the normative model: $54.3 \%-54.8 \%$ for the reference point probability and $1.6 \%$ to $1.9 \%$ for the other component. The proportions predicted by the Nilsson et al. model depend on the weights assumed for the component probabilities ${ }^{\mathrm{iv}}$. In its standard form, the weights are 0.8 for the reference point probability and 0.2 for the other component probability. Using these weights, a simulation revealed that the proportion of variance accounted for ranged between $60.8 \%$ and 61.3 for the reference point probability and $3.7 \%$ to $3.9 \%$ for the other component.

While the normative and these other two models assume that, at the level of the individual, the joint probability is some linear combination of the component probabilities, our view point is different. We argue that the presence of a minority of participants who, contrary to expectation, set the conjunctive (disjunctive) probability exactly equal to the more (less) likely component event may be symptomatic of a somewhat larger group who also based their judgement solely on the more (less) likely component, if not exactly equal to it. With a majority of participants basing their judgement on just one component and the remainder basing it solely on the other, this will render both components statistically significant in the MLM analyses of the joint event probabilities.

When we analysed the 12 conjunctive probability estimates produced by each participant (i.e., one regression for each participant), with the more likely and less likely component probabilities as 
IVs, for those cases where significant effects were observed, in 27 cases only the less likely component was significant with only the more likely in a further 3 cases. Only 5 participants produced estimates such that both components played a statistically significant role. The regressions with the disjunctive probability as DV also produced a similar pattern with just 7 participants producing estimates where both components were statistically significant. For the remainder, only the more likely event was significant in 11 cases, and only the less likely in 12 cases. It is noteworthy that the less likely event appears to serve as the reference point for the disjunction as often as the more likely component. This outcome is consistent with the fact that the proportion of cases where the disjunctive probability was set exactly equal to the more likely component was similar to the proportion where it was set exactly equal to the less likely component. While our results are broadly consistent with proposition 1, it must be acknowledged that the choice of reference point may be atypical for a minority of participants, more (less) likely in the conjunctive (disjunctive) case.

As with Study 1 and consistent with proposition 3, overall our results suggest that there was no statistically significant relationship between the conjunctive-less likely probability difference and the more likely-disjunctive probability difference. With regard to the role of the component probabilities in determining the conjunctive-less likely probability difference and the more likelydisjunctive probability difference, for those individuals avoiding the fallacy, the results were exactly in line with our expectations in that only the less (more) likely event was statistically significant as a predictor in the conjunctive-less likely (more likely-disjunctive) case. For those committing the fallacy both components were statistically significant. However, here we would again argue that this outcome was due to the minority of participants who selected the other component (more likely in the conjunctive case and less likely in the disjunctive) as the reference point. When taken together with the majority who behaved as predicted, this would produce the pattern of results that was observed.

\section{GENERAL DISCUSSION}

Considering the outcomes of Studies 1 and 2, we contend that the key assumptions and expectations underpinning our model have been upheld. A key aspect of our theoretical position is that only one component influences the estimation of the joint probability with the other one exerting no direct influence in this regard. This being the case, it might be expected that a minority of participants would actually set the joint event probability exactly equal to the relevant component probability and we found this to be the case. Indeed, in both studies this was the modal response with the disjunctive set exactly equal to the more likely component probability and the conjunctive to the less likely component probability. We are not the first to observe this tendency. Indeed, regarding conjunctive judgements, it was a common response among Hertwig and Chase's (1998) participants. Similarly, many of Biela's (1986) participants set the disjunctive probability exactly equal to the more likely event. Well before the conjunction fallacy phenomenon was popularised a radically different 
account of subjective probability judgement was put forward (Shackle, 1969). Central to this account is the proposition that it is the capacity of events to surprise us which determines the probabilities we assign to them, the more surprising an event the less likely it is deemed to be. According to this 'potential surprise' framework, joint events, conjunctive and disjunctive, also possess surprise value but surprise is determined solely with reference to the more surprising event in the conjunctive case and the less surprising event in the disjunctive. In other words, the surprise value of joint events is not determined by any additive or multiplicative rule but rather by a MIN/MAX rule. In this sense, potential surprise makes the same predictions as our own model and may provide an explanation as to why the modal response in both our studies was to set the joint event exactly equal to the reference point.

More generally, the results of Study 1 are, almost without exception, consistent with our predictions. Only the less (more) likely event accounted for statistically significant variance in the conjunctive (disjunctive) probability (proposition 1). We also predicted that the conjunctive-less likely and the more likely-disjunctive probability differences would be predicted solely by the less and more likely component probabilities respectively and for the most part this was borne out (proposition 2). Furthermore, the conjunctive- less likely and the more likely-disjunctive differences were uncorrelated (proposition 3).

As we previously noted, most accounts of the conjunction fallacy, either directly or indirectly, posit a role for the more likely component event in somehow distorting the probability assigned to the conjunctive event, leading to the conjunction fallacy (Busemeyer et al 2011; Epstein et al. 1995; Tversky \& Kahneman, 1983; Wells, 1985; Yates \& Carlson, 1986). Tentori et al. (2013) make almost exactly the same point in developing their theory of inductive confirmation:

'The accounts listed in the foregoing pages involve a variety of quite different assumptions and implications concerning human judgment. Yet all of them-along with others that have not been included for the sake of brevity .... converge on the widely shared view that the perceived probability of the added conjunct [usually the more likely event] is a crucial factor governing the occurrence of conjunction fallacy effects. This means that in otherwise controlled conditions, the more probable the added conjunct, the stronger the conjunction fallacy effect to be expected.' (Tentori et al, 2013, p239, italics added by the present authors)

Clearly, at face value, our results are not consistent with the view prevailing in much of the relevant experimental literature. In study 1 , the likely event was not statistically significant as a predictor of the conjunctive event. Furthermore, in Study 2, while both components were statistically significant, the less likely component event accounted for $41 \%$ of the variance in the conjunctive probability with the more likely event accounting for just $6 \%$.

It appears that virtually all outcomes were in accordance with prediction in Study 1 and if there were a minority of participants selecting the other component as the reference point then they were not sufficient in number to affect the results. However, some aspects of the results from Study 2 
require further exploration. In the MLM analyses, while both component probabilities were statistically significant in determining the conjunctive and disjunctive probabilities, we argue that this is due to the fact that a minority of participants adopt the other component as their reference point while the majority do base their judgement on the predicted component (less likely in the conjunctive case, more likely in the disjunctive). In support of this proposition, for both conjunctive and disjunctive judgements, we identified a number of participants who actually set the joint event probability exactly equal to one or other component, usually in accordance with our expectation but sometimes selecting the other component. We argue that this may be a reflection of a wider tendency, among a minority of participants, to base the conjunctive (disjunctive) probability on the more (less) likely component. Indeed, when we ran regressions for individual participants we found that, for the most part, only one component was statistically significant in predicting the joint probability, usually the predicted one but sometimes the other component, especially in the case of the disjunctive judgement where a noticeable minority appeared to select the less likely component. Overall, we found little evidence to suggest that individuals form their conjunctive or disjunctive probability estimates as a weighted average of the two component probabilities.

With regard to the conjunctive-less likely and the disjunctive-more likely probability differences, the normative model requires that these two quantities are identical (see Equation 10) and by implication perfectly correlated. Furthermore, when expressed as a linear function with the difference as DV and the components as IVs, given the normative relationship, it can be shown that both components probabilities should play an approximately equal role in determining the joint eventcomponent probability difference ${ }^{\mathrm{v}}$. This contrasts with the results we present in Table 1 and Table 5 . In Study 1, with only one minor exception, only the less (more) likely component was statistically significant in predicting the conjunctive-less likely (more likely-disjunctive) difference. For those avoiding the fallacy, the same outcome emerged in Study 2. While it might be argued that those participants committing the fallacy in Study 2 did, in a sense, behave normatively (since for them both components were statistically significant), this argument is difficult to sustain since these were the participants who breached the normative rule by actually committing the fallacy. More decisively, we found that in both Studies 1 and 2, the conjunctive-less likely and the disjunctive-more likely differences were not significantly correlated which contrasts with the normative situation in which the two quantities are identical (see Equation 10).

Interestingly, it can be shown that under standard conditions in both Costello and Watts' (2016) model (in which judgements are essentially normative but subject to systematic but mutually self-cancelling errors) and Nilsson and co-workers' (2009) model (where joint probabilities are said to be weighted averages of the component probabilities), the two joint event-component probability differences (conjunctive-less likely, more likely - disjunctive) should be correlated ${ }^{\mathrm{vi}}$. From the results that we present in Studies 1 and 2 we see that this was not the case. The absence of such a correlation is consistent with our own model where the statistical significance of each IV reflects the choice of 
different reference points among our participants. For the majority, the reference point is the less likely component in the conjunctive case and the more likely component in the disjunctive. For the minority the opposite tendency is manifested. However, as long as the size of this minority is limited and/or their choice of reference point is symmetrical (i.e., more likely in the conjunctive and less likely in the disjunctive case), then at the aggregate level, both components will potentially be significant in determining both the joint event probabilities and hence joint event-component probability differences, while the differences themselves will be uncorrelated. Having said this it is important to note that the models of both Costello and Watts and Nilsson et al. both assume the presence of random errors in the process through which individuals derive their joint event probabilities. This being the case it must be acknowledged that where these errors are sufficiently large the outcomes predicted by their models are broadly consistent with the results we report here.

An aspect of our model that we have so far neglected is the nature of the quasi random adjustment from the assumed reference point. While we maintain that the magnitude of the adjustment is quasi random, what we mean by this is that its magnitude appears to be unrelated to the other component probability and as yet is not related to any known influence. However, in terms of the direction, our results reveal that this aspect is not random. Specifically, if a participant made negative adjustments in one subset of scenarios, for example, thereby avoiding (committing) the fallacy in the conjunctive (disjunctive) case he/she is more likely to make negative adjustments in the remaining scenarios. It is possible that some of the mechanisms previously put forward to account for the conjunction fallacy might be manifested as quasi random adjustments. For example, Yates and Carlson (1986) asked their participants to provide verbal accounts of the strategies they used in forming their conjunctive judgements. It is possible that a number of the strategies that were forthcoming may manifest themselves as qualitative all or nothing adjustments. For example, some individuals appeared to possess a basic understanding of the extension principle, without being aware of the actual probabilistic rule. These individuals knew that the conjunction could not be more likely than the less likely component but were unable to express this knowledge in precise numerical terms. Thus, it is possible that this understanding was manifested as a quasi-random negative adjustment from the reference point. Another common strategy identified by Yates and Carlson (1986) was 'substantive reasoning'. Here their participants tended to go beyond the information provided and develop additional arguments and assumptions to justify their decision to rank $\mathrm{P}(\mathrm{A} \& \mathrm{~B})>\mathrm{P}(\mathrm{B})$. Clearly such assumptions may be qualitative in nature and go beyond the potential influence that the component probabilities may exert. As such, they might be manifested as quasi-random adjustments. For those influenced by the representativeness heuristic, in those cases where the evidence is representative of the more likely event then this might result in a positive quasi random adjustment from the less likely event giving rise to the conjunction fallacy. Crucially, it may be that the magnitude of this adjustment is unrelated to the actual degree of representativeness and that all that is necessary is that the degree of representativeness exceeds some threshold value. The same might be 
said in relation to events that are inductively confirmed by the evidence (Tentori et al.). As we note above, our results show that those who tend to make a positive adjustment in one scenario are more likely to do so in the remaining scenarios. In relation to our second stage of the judgement process, this is perhaps indicative of the consistent application of some strategy, for example representativeness, across a range of judgements.

We previously argued that for normative judgements, the less likely event probability accounted for more variance in the conjunctive probability compared to the more likely component event. This can be demonstrated mathematically and statistically ${ }^{\text {vii }}$ but in more simple terms, a 0.1 increase (decrease) in the less likely probability will give rise to a larger increase (decrease) in the conjunctive probability than a 0.1 unit increase (decrease) in the more likely probability. Keeping this in mind, in the case of the disjunctive probability, examination of equation 6 reveals that most of any increase in the less likely probability will feed through to the conjunctive probability effectively cancelling most of this increase out leaving the disjunctive probability only marginally larger. As we have argued above, the equivalent feed through effect of the more likely probability results in a smaller change in the conjunctive probability. Thus, most of the increase in the more likely probability will be manifested in the disjunctive probability. The implication of this is that a 0.1 increase in the more likely probability will have a greater effect on the disjunctive probability than a 0.1 increase in the less likely probability. In this sense, our model appears to produce results which mirror aspects of normative probability judgement in that we have shown that the less (more) likely event accounts for most of the variance in the conjunctive (disjunctive) event probability. This presents a rather positive view of probability judgement in that while estimates of joint probability may be fallacious and thus conceptually flawed, nonetheless they may be close enough to the normative values to allow decisions, if not perfect, to be good enough. Indeed, where information is imperfect, conditions are changeable, where rapid decisions are needed and cognitive resources are limited, focussing on the less (more) likely event in the conjunctive (disjunctive) context may be the only practical strategy.

An issue arising from our analytical approach is whether our theoretical position is falsifiable. We acknowledge that for a single judgement by one individual our model makes predictions that cannot be falsified since the reference point probability is not invariably less likely for conjunctive and more likely for disjunctive and the quasi-random adjustment can, in either case, be positive or negative. However, the underlying assumption of our model is that individuals become aware of the respective roles played by the components in determining the joint event probabilities through the accumulated experience of every day events and combinations of events. Since normative relationships underpin real world events, experience will dictate that it is the less likely event that is key in the conjunctive case and the more likely in the disjunctive case. While this knowledge may be incomplete (for example, in the sense that the role of the other component remains opaque), it is reasonable to assume that the majority of participants will, in broad terms, respond in accordance with 
these basic learned relationships. In other words, we are arguing that this will be the dominant tendency. Therefore, a randomly selected sample of appropriate size would be expected to result in the less (more) likely event probability accounting for most of the variance in the conjunctive (disjunctive) probability. Thus, in future research, the proportions of unique variance accounted for by each component event could be compared statistically for each sample and the results over a number of samples could be analysed using meta analytical techniques. In this way, it would be possible to determine whether the proportion associated with a given component was significantly larger than that of the other component. Were it the case that there was no significant difference then the theory would be falsified.

We realise that various of our predictions follow from other accounts of the fallacy including the normative model (if a random error component is incorporated) as well as in the models of Costello and Watts $(2014 ; 2016)$ and Nilsson et al. (2009). However, in their standard form all these other models predict a significant relationship between the conjunctive-less likely and the more likely disjunctive difference, whereas we do not. In our model, these differences are neither correlated nor will the two quantities necessarily be equal (as they would be expected to be in the other models). We do however concede that failure to reject the null hypothesis does not constitute proof for it. Equally while it may be technically possible within our model that that a substantial number of individuals base both joint events on the same component, since we believe most of the variance in the adjustment to be random, even in this extreme situation we would not predict a significant relationship between the two differences. Should a situation arise where the data reliably demonstrate that the conjunctive-less likely and the more likely disjunctive differences are significantly correlated then we would consider our model falsified (at the very least in the sense that it cannot be differentiated from the other models).

To conclude, we contend that the results we present here are consistent with the model that we propose. In making probability judgements for joint events, individuals appear to first select one of the components as a reference point, usually but not invariably, the less likely in the conjunctive case and the more likely in the disjunctive. In some instances, the joint event probability is set exactly equal to the reference point. Otherwise, we propose that a quasi-random adjustment, positive or negative, is made in the second stage of the judgement. We believe that the direction of this adjustment will be determined by the application of some qualitative process which may sometimes result in fallacious reasoning. Our model generates a number of predictions concerning the relationship between the joint probabilities and the components and between the conjunctive-less likely and the more likely-disjunctive differences. Overall, our results are consistent with these predictions.

\section{Declaration of Interest}


None of the authors have any interests to declare. No external funding was received. 
Armantier O. (2006). Estimates of own lethal risks and anchoring effects. Journal of Risk and Uncertainty, 32, 37-56

Biela, A. (1986). Psychological patterns in predicting disjunction and conjunction of clinical symptoms. Acta Psychologica, 61, 183-195.

Bar-Hillel, M., and Neter, E. (1993). How alike is it versus how likely is it: A disjunction fallacy in probability judgements. Journal of Personality and Social Psychology, 65, 1119-1131.

Busemeyer, J. R., Pothos, E. M., Franco, R., \& Trueblood, J. S. (2011). A quantum theoretical explanation for probability judgment errors. Psychological Review, 118(2), 193-218. doi:10.1037/a0022542

Carlson, B.W. and Yates, J.F. (1989). Disjunction errors in qualitative likelihood judgement. Organizational Behavior and Human Decision Processes, 44, 368-379.

Carpenter, A. C., \& Schacter, D. L. (2017). Flexible retrieval: When true inferences produce false memories. Journal of Experimental Psychology: Learning, Memory, and Cognition, 43, 335349. doi:10.1037/xlm0000340

Combs B., and Slovic, P. (1979). Newspaper Coverage of Causes of Death. Journalism Quarterly, 56, 837-843.

Costello, F., \& Watts, P. (2014). Surprisingly rational: Probability theory plus noise explains biases in judgment. Psychological Review, 121(3), 463-480. doi:10.1037/a0037010

Costello, F., \& Watts, P. (2016). Explaining high conjunction fallacy rates: the probability theory plus noise account. Journal of Behavioral Decision Making, Published online in Wiley Online Library (wileyonlinelibrary.com) DOI: 10.1002/bdm.1936

Devitt, A. L., Monk-Fromont, E., Schacter, D. L., \& Addis, D. R. (2016). Factors that influence the generation of autobiographical memory conjunction errors. Memory, 24(2), 204-222. doi: $10.1080 / 09658211.2014 .998680$

Epstein, S., Denes-Raj, V., \& Pacini, R. (1995). The Linda problem revisited from the perspective of cognitive-experiential self-theory. Personality and Social Psychology Bulletin, 21, 11241138. doi:10.1177/01461672952111001

Fiedler, K. (1988). The dependence of the conjunction fallacy on subtle linguistic factors.

Psychological Research, 50, 123-129.

Gavanski, I. and Roskos-Ewoldsen, D.R. (1991) Representativeness and conjoint probability, Journal of Personality and Social Psychology, 61: 181-194.

Hakes, J.K., \& Viscusi, W.K. (2004). Dead reckoning: Demographic determinants of the accuracy of mortality risk perceptions. Risk Analysis, 24, 651-664.

Hertwig, R., \& Chase, V. M. (1998). Many reasons or just one: How response mode affects reasoning in the conjunction problem. Thinking and Reasoning, 4, 319-352.Hyman, I. J., \& Loftus, E. F. (1998). Errors in autobiographical memory. Clinical Psychology Review, 18(8), 933-947. doi:10.1016/S0272-7358(98)00041-5 
Hyman Jr., I.E. and Loftus, E.F. (1998) Errors in autobiographical memory. Clinical Psychology Review, 18, 933-947.

Kahneman, D. (2016). Heuristics and biases. In R. J. Sternberg, S. T. Fiske, D. J. Foss, R. J. Sternberg, S. T. Fiske, D. J. Foss (Eds.), Scientists making a difference: One hundred eminent behavioral and brain scientists talk about their most important contributions (pp. 171-174). New York, NY, US: Cambridge University Press.

Kariyazono, A. (1991). The study of conjunction fallacy in the probability judgment task of compound event. Japanese Journal of Psychonomic Science, 10, 57-64. (English Abstract.)

Lichtenstein, S., Slovic, P., Fischhoff, B., Layman, M., \& Combs, B. (1978). Judged frequency of lethal events. Journal of Experimental Psychology: Human Learning and Memory, 4(6), 551578. doi:10.1037/0278-7393.4.6.551

Lu, Y. (2016). The conjunction and disjunction fallacies: Explanations of the Linda problem by the Equate-to-Differentiate model. Integrative Psychological and Behavioral Science, 50, 507531.

Nilsson, H., Winman, A., Juslin, P., \& Hansson, G. (2009). Linda is not a bearded lady: Configural weighting and adding as the cause of extension errors. Journal of Experimental Psychology: General, 138(4), 517-534. doi:10.1037/a0017351

Odegard, T. N., \& Lampinen, J. M. (2004). Memory conjunction errors for autobiographical events: More than just familiarity. Memory, 12(3), 288-300. doi:10.1080/09658210244000621

Shackle, G. L. S. (1969). Decision, Order and Time in Human Affairs. Cambridge: Cambridge University Press.

Tentori, K., Crupi, V., \& Russo, S. (2013). On the determinants of the conjunction fallacy: Probability versus inductive confirmation. Journal of Experimental Psychology: General, 142, 235-255.

Thuring, M. \& Jungermann H. (1990) The conjunction fallacy: Causality versus event probability. Journal of Behavioral Decision Making, 3, 61-74

Tversky, A., \& Kahneman, D. (1983). Extensional versus intuitive reasoning: The conjunction fallacy in probability judgment. Psychological Review, 90, 293-315.

Wells, G.L. (1985) The conjunction error and the representativeness heuristic, Social Cognition, 3: 266-279.

Wyer, R. S. (1970). Quantitative prediction of belief and opinion change: A further test of a subjective probability model. Journal Of Personality And Social Psychology, 16(4), 559-570. doi: $10.1037 / \mathrm{h} 0030064$

Xu, R. (2003). Measuring explained variation in linear mixed effects models. Statistics in Medicine, 22, 3527-3541. DOI: $10.1002 / \operatorname{sim} .1572$

Yates, J.F., \& Carlson, B.W. (1986). Conjunction errors: Evidence for multiple judgment procedures including "Signed Summation". Organizational Behavior and Human Decision Processes, $37,230-253$. 
Table 1. The Role of the More and Less Likely Component Probabilities in Determining the Joint Event Probabilities and the Joint Event-Component Probability Differences: Study 1

\begin{tabular}{|c|c|c|c|c|c|c|c|c|c|}
\hline \multirow[t]{2}{*}{ Dependent Variable } & \multicolumn{3}{|c|}{ Intercept } & \multicolumn{3}{|c|}{ More Likely Probability } & \multicolumn{3}{|c|}{ Less Likely Probability } \\
\hline & beta & $\mathrm{t}$ & $\mathrm{p}$ & beta & $\mathrm{t}$ & $\mathrm{p}$ & beta & $\mathrm{t}$ & $\mathrm{p}$ \\
\hline & \multicolumn{9}{|c|}{ Person Based Scenarios } \\
\hline All judgements & & & & & & & & & \\
\hline $\begin{array}{l}\text { Conjunctive } \\
\text { probability } \\
\text { estimate }\end{array}$ & 16.98 & 5.12 & $<.001$ & 0.027 & 0.60 & .549 & 0.624 & 11.46 & $<.001$ \\
\hline $\begin{array}{l}\text { Inclusive } \\
\text { Disjunctive } \\
\text { probability } \\
\text { estimate }\end{array}$ & 51.81 & 14.62 & $<.001$ & 0.125 & 3.26 & .002 & 0.028 & 0.71 & .478 \\
\hline \multirow{3}{*}{\multicolumn{10}{|c|}{$\begin{array}{l}\text { Judgements such that } \\
\mathrm{P}(\mathrm{AB}) \neq \mathrm{P}(\mathrm{A}) \text { and } \\
\mathrm{P}(\mathrm{AB}) \neq \mathrm{P}(\mathrm{B})\end{array}$}} \\
\hline & & & & & & & & & \\
\hline & & & & & & & & & \\
\hline $\begin{array}{l}\text { Conjunctive } \\
\text { probability } \\
\text { estimate }\end{array}$ & 24.89 & 5.54 & $<.001$ & -0.027 & -0.49 & .627 & 0.504 & 6.48 & $<.001$ \\
\hline $\begin{array}{l}\text { Inclusive } \\
\text { Disjunctive } \\
\text { probability } \\
\text { estimate }\end{array}$ & 50.807 & 12.66 & $<.001$ & 0.158 & 4.27 & $<.001$ & 0.009 & 0.21 & .838 \\
\hline \multicolumn{10}{|l|}{$\begin{array}{l}\text { Conjunctive } \\
\text { probability minus } \\
\text { Less Likely }\end{array}$} \\
\hline Main Effect ${ }^{1}$ & -4.837 & -1.10 & .272 & 0.046 & 0.64 & .522 & -0.359 & -4.62 & $<.001$ \\
\hline $\begin{array}{l}\text { Fallacy } \\
\text { Interaction }\end{array}$ & 27.517 & 5.27 & $<.001$ & 0.046 & 0.55 & .583 & 0.120 & 1.26 & .207 \\
\hline \multicolumn{10}{|l|}{$\begin{array}{l}\text { More likely minus } \\
\text { Disjunctive } \\
\text { probability }\end{array}$} \\
\hline Main Effect ${ }^{1}$ & -44.537 & -7.32 & $<.001$ & 0.473 & 5.52 & $<.001$ & -0.010 & -0.14 & .893 \\
\hline $\begin{array}{l}\text { Fallacy } \\
\text { Interaction }\end{array}$ & 25.213 & 2.95 & .004 & 0.126 & 1.06 & .294 & -0.057 & -0.56 & .575 \\
\hline Main Effect ${ }^{2}$ & -19.324 & -2.53 & .013 & 0.599 & 5.83 & $<.001$ & -0.066 & -0.94 & .347 \\
\hline
\end{tabular}


Table 2. The Relationship Between the Conjunctive and Disjunctive Probability Estimates ${ }^{\mathrm{a}}$ :Study 1

\begin{tabular}{|c|c|c|c|c|c|c|c|c|c|c|}
\hline \multirow[t]{2}{*}{ Scenario } & \multicolumn{2}{|c|}{ More Likely } & \multicolumn{2}{|c|}{ Less Likely } & \multicolumn{2}{|c|}{ Conjunction } & \multicolumn{2}{|c|}{ Disjunction } & \multicolumn{2}{|c|}{$\begin{array}{l}\text { Conj.-Disj. } \\
\text { Correlation }\end{array}$} \\
\hline & Mean & SD & Mean & SD & Mean & SD & Mean & SD & $\mathrm{r}$ & $\mathrm{p}$ \\
\hline Ollie & 71.80 & 21.20 & 30.63 & 20.63 & 37.95 & 28.05 & 62.40 & 22.76 & .113 & .486 \\
\hline Linda & 68.75 & 23.22 & 34.50 & 21.89 & 40.33 & 24.09 & 61.90 & 23.49 & .135 & .407 \\
\hline Bill & 66.00 & 21.70 & 25.33 & 19.16 & 30.85 & 20.57 & 61.38 & 23.56 & .039 & .813 \\
\hline Tom & 47.38 & 15.32 & 37.90 & 18.28 & 39.60 & 19.04 & 58.03 & 20.19 & .272 & .089 \\
\hline Rick & 46.85 & 15.46 & 39.48 & 17.80 & 40.95 & 21.70 & 58.93 & 19.69 & .402 & .010 \\
\hline
\end{tabular}

a: for those participants in the inclusive disjunction condition. 
Table 3. Relationship Between the Joint Event-Component Probability Differences ${ }^{\mathrm{a}}$ : Study 1

\begin{tabular}{lrrrrrrrr}
\hline \multirow{2}{*}{ Scenario } & \multicolumn{2}{c}{ Conjunctive- Less } & \multicolumn{2}{c}{ More - Disjunctive } & \multicolumn{2}{c}{ Correlation } & \multicolumn{2}{c}{ Difference } \\
\cline { 2 - 9 } & Mean & SD & Mean & SD & r & p & t & $\mathrm{p}$ \\
\hline Ollie & 7.33 & 26.97 & 9.40 & 24.12 & -.222 & .168 & -0.33 & .744 \\
Linda & 5.83 & 20.46 & 6.85 & 26.25 & -.219 & .175 & -0.18 & .860 \\
Bill & 5.53 & 19.84 & 4.63 & 24.08 & -.004 & .980 & 0.18 & .856 \\
Tom & 1.70 & 11.21 & -10.65 & 21.25 & .249 & .121 & 3.65 & $<.001$ \\
Rick & 1.48 & 13.07 & -12.08 & 20.34 & -.223 & .166 & 3.23 & .003 \\
\hline
\end{tabular}

a: for those participants in the inclusive disjunction condition. 
Table 4. The Relationship Between the Component Probability Estimates ${ }^{\mathrm{a}}$ : Study 2

\begin{tabular}{|c|l|l|l|l|l|l|}
\hline $\begin{array}{l}\text { Weather Joint } \\
\text { Event }\end{array}$ & \multicolumn{2}{l}{$\begin{array}{l}\text { More-Less } \\
\text { Likely } \\
\text { Correlation }\end{array}$} & \multicolumn{2}{l|}{$\begin{array}{l}\text { More } \\
\text { Event }\end{array}$} & \multicolumn{2}{l|}{$\begin{array}{l}\text { Less Likely } \\
\text { Event }\end{array}$} \\
\hline & $\mathrm{r}$ & $\mathrm{p}$ & Mean & SD & Mean & SD \\
\hline Cold and/or & & & & & & \\
\hline Sunny & .021 & .872 & 71.93 & 20.43 & 35.38 & 17.69 \\
\hline Thundery & .145 & .270 & 70.83 & 21.38 & 18.58 & 16.21 \\
\hline Windy & .724 & $<.001$ & 72.72 & 20.58 & 55.03 & 23.57 \\
\hline Cloudy & .595 & $<.001$ & 73.90 & 19.53 & 57.46 & 21.31 \\
\hline Frosty and/or & & & & & & \\
\hline Sunny & .356 & .005 & 43.10 & 17.92 & 23.05 & 13.08 \\
\hline Thundery & .673 & $<.001$ & 31.62 & 17.93 & 16.63 & 14.50 \\
\hline Windy & .528 & $<.001$ & 58.77 & 22.03 & 27.82 & 17.54 \\
\hline Cloudy & .387 & .002 & 61.45 & 20.14 & 28.75 & 17.81 \\
\hline Sleety and/or & & & & & & \\
\hline Sunny & .446 & $<.001$ & 41.02 & 18.48 & 17.15 & 11.24 \\
\hline Thundery & .716 & $<.001$ & 26.15 & 18.38 & 14.12 & 12.24 \\
\hline Windy & .370 & .004 & 57.32 & 23.67 & 21.28 & 16.93 \\
\hline Cloudy & .331 & .010 & 61.93 & 20.14 & 20.28 & 15.33 \\
\hline
\end{tabular}


Table 5. The Role of the More and Less Likely Component Probabilities in Determining the Joint Event Probabilities and the Joint Event-Component Probability Differences: Study 2

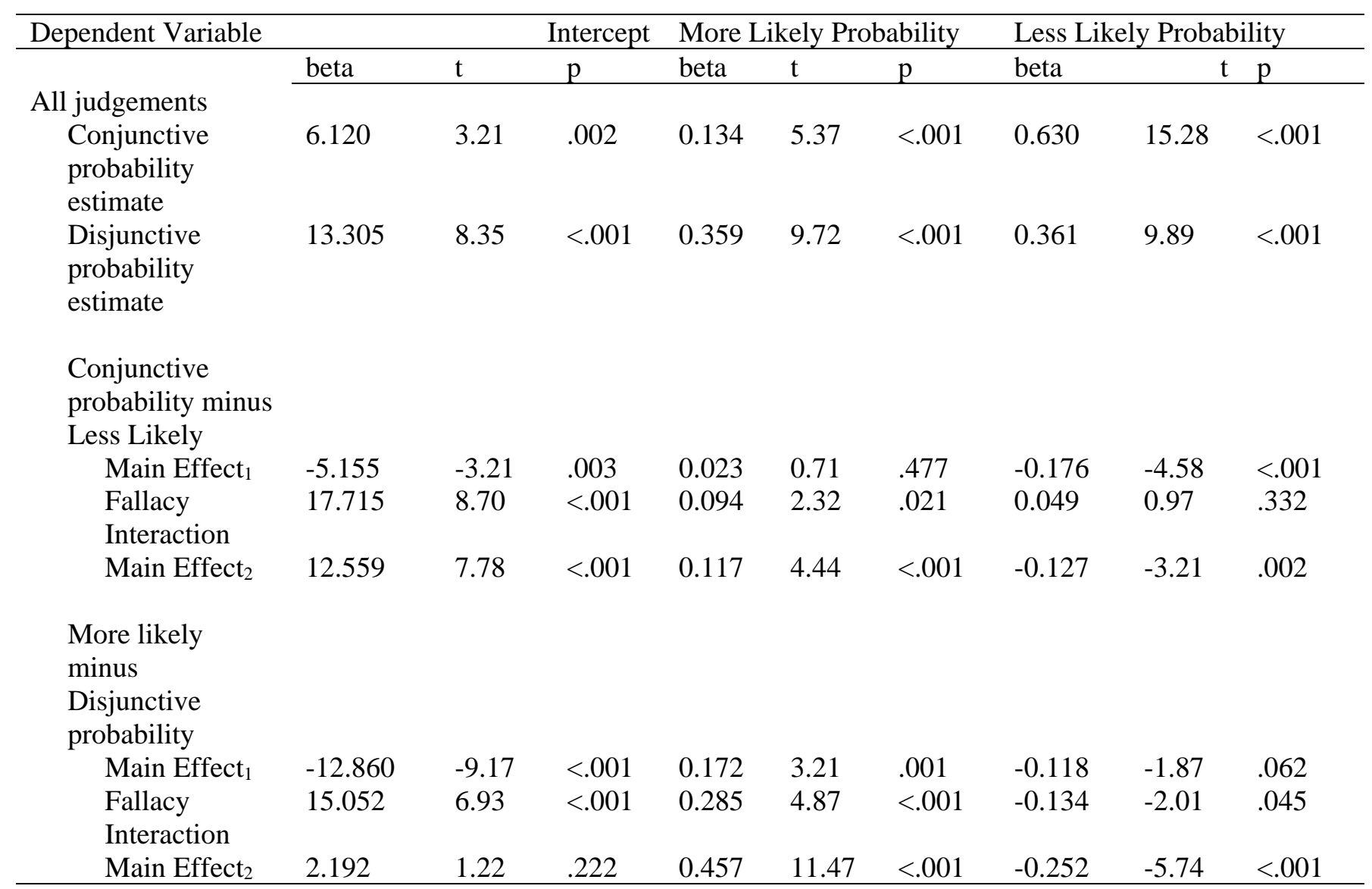


Table 6. The Relationship Between the Joint Event-Component Probability Differences ${ }^{\mathrm{a}}$ : Study 2

\begin{tabular}{|c|c|c|c|c|c|c|c|c|}
\hline \multirow{2}{*}{$\begin{array}{l}\text { Weather } \\
\text { Combination }\end{array}$} & \multicolumn{2}{|c|}{ Conjunctive- Less } & \multicolumn{2}{|c|}{ More - Disjunctive } & \multicolumn{2}{|c|}{ Correlation } & \multicolumn{2}{|c|}{ Difference } \\
\hline & Mean & SD & Mean & SD & $\mathrm{r}$ & $\mathrm{p}$ & $\mathrm{t}$ & $\mathrm{p}$ \\
\hline 1 & 5.87 & 16.78 & 1.23 & 16.10 & -.155 & .237 & 1.44 & .156 \\
\hline 2 & 5.55 & 16.07 & 3.20 & 17.08 & .065 & .621 & 0.80 & .425 \\
\hline 3 & 0.45 & 17.64 & 6.17 & 21.95 & .277 & .032 & -1.84 & .071 \\
\hline 4 & -1.73 & 18.11 & 0.93 & 25.26 & .050 & .706 & -0.68 & .499 \\
\hline 5 & 8.47 & 23.14 & 12.60 & 21.75 & -.024 & .856 & -1.00 & .323 \\
\hline 6 & 6.12 & 19.89 & 19.42 & 24.53 & -.045 & .731 & -3.19 & .002 \\
\hline 7 & 4.22 & 12.20 & 27.93 & 25.93 & .345 & .007 & -7.48 & $<.001$ \\
\hline 8 & 6.27 & 16.11 & 21.05 & 25.59 & .009 & .945 & -3.80 & $<.001$ \\
\hline 9 & 8.12 & 15.23 & 9.40 & 23.94 & .008 & .951 & -0.35 & .726 \\
\hline 10 & 4.90 & 23.82 & 13.88 & 26.50 & -.040 & .763 & -1.92 & .060 \\
\hline 11 & 0.10 & 19.32 & 11.67 & 18.90 & -.001 & .994 & -3.31 & .002 \\
\hline 12 & -1.27 & 16.38 & 11.35 & 21.26 & .041 & .755 & -3.72 & $<.001$ \\
\hline
\end{tabular}


i Given the normative relationship: 
$P(A \& B)=P(B) X P(A \mid B)$ and given $P(B)<P(A \mid B)$

Let $y=$ some arbitrary constant such that $y<=1-P(A \mid B)$ (this condition is necessary to ensure that $P(A \mid B)+y$ $<=1$

Examining the effects on the conjunctive probability of an increase in the value of each component by this constant $y$ we see that for the less likely event:

$P(B+y) \times P(A \mid B)=P(A \& B)+y X P(B \mid A)$

For the more likely event an increase by the constant $\mathrm{y}$ is such that

$P(B) X P(A \mid B+y)=P(A \& B)+y X(P(B)$

Since $P(B)<P(A \mid B)$ it follows that

$Y X P(B)<y X P(A \mid B)$

Therefore $P(B+y) \times P(A \mid B)>P(B) X(A \mid B+y)$

So the effect on the conjunctive probability of an increase in each component probability by some constant $y$ is greater for the less likely event. Hence arbitrary variation in the less likely probability has a greater effect on the conjunctive probability compared to similar variation in the more likely event and therefore, other things being equal, in the normative case the less likely event will account for most of the variance in the conjunctive event.

Given $P(B)<P(A)$ and the normative disjunctive relationship:

$P(A$ or $B)=P(A)+P(B)-P(A \& B)$

In relation to the first part of the expression:

$P(A)+P(B)$

Clearly an increase of $y$ in either component will have the same effect. However, given the negative role played by the conjunctive event $P(A \& B)$ in the above expression, we have already demonstrated that an increase of the $y$ in the smaller component probability will give rise to a larger increase in the conjunctive event relative to the same increase in the more likely event. Therefore, most of the effect of an increase of $y$ in $P(B)$ will feed through to the conjunctive event thereby negating the effect of the increase. This is less so in the case of an increase of $y$ in the more likely event.

ii We consider the adjustment to be random because given our previous conjunction fallacy research the magnitude of the adjustment appears to be unrelated to any of the relevant variables we have considered, most importantly, the more likely component. It may be that in future research, factors influencing the magnitude of the adjustment may be identified. For example, recently, in as yet unpublished research, we have examined the extent to which the degree of inductive confirmation associated with the more likely component (Tentori et al. 2013) might affect the conjunctive probability. However again we found no direct relationship. We acknowledge that the adjustment is quasi random in the sense that it cannot give rise to probabilities which exceed unity or which are less than zero. In that sense in the conjunctive case it is bounded by the interval between the less likely event and either the zero point on the probability continuum (negative adjustment) or by the unity point (positive adjustment).

iii For example, for the component probabilities, $P_{E}(A)=\mathrm{P}(\mathrm{A})+\mathrm{d}-2 \mathrm{dP}(\mathrm{A})$, where $P_{E}(A)$ is the estimated probability and $\mathrm{P}(\mathrm{A})$ is the objective probability.

iv The Nilsson et al model predicts that joint probabilities are derived from the component event probabilities in a manner consistent with the two equations below, where the subscripts ${ }_{L}$ and $_{H}$ refer to the component with the lower or higher probability value: 
$P\left(A_{L} \& B_{H}\right)=(1-b) \times P\left(A_{L}\right)+b \times P\left(B_{H}\right)$

$P\left(A_{L}\right.$ or $\left.B_{H}\right)=b^{\prime} \times P\left(A_{L}\right)+\left(1-b^{\prime}\right) \times P\left(B_{H}\right)$

The parameters, $b$ and $b^{\prime}$, determine the weights assigned to the component probabilities in determining the joint event probabilities. Nilsson et al. (2009) suggest that, in general, $0 \geq b \leq 0.5$ although in their experimental studies they assumed a value for $b=0.2$. The same assumptions apply to $b^{\prime}$

${ }^{v}$ Simulations demonstrating this are available from the corresponding author.

${ }^{v i} A$ proof of this assertion is available from the corresponding author

vii A simulation is available from the corresponding author and see footnote (i). 